\title{
Wajir, Kenya: Adult COVID-19 perceptions, prevention practices and impact-Responses from the first round of adult data collection in Wajir, Kenya
}

Population Council

Follow this and additional works at: https://knowledgecommons.popcouncil.org/departments_sbsr-pgy How does access to this work benefit you? Let us know!

\section{Recommended Citation}

"Wajir, Kenya: Adult COVID-19 perceptions, prevention practices and impact-Responses from the first round of adult data collection in Wajir, Kenya," COVID-19 Research \& Evaluations brief. Nairobi: Population Council, 2020. 
Ideas. Evidence. Impact.

\section{Wajir, Kenya: Adult COVID-19 Perceptions, Prevention Practices and Impact \\ Responses from the first round of adult data collection in Wajir, Kenya}

July 2020

\section{Highlights}

- Overall, COVID-19 knowledge in Wajir is inadequate and adoption of prevention behaviors could be strengthened. 2 in 5 respondents (39\%) do not know that anyone can be infected with COVID19 and $32 \%$ believe the myth that COVID-19 just a common cold. Only $54 \%$ of respondents wore a mask in the past week when leaving their homes.

- Fear of beingstigmatized if infected with COVID-19 is pervasive: most respondents (62\%) believe people would stop talking to them if they became infected and less than half (42\%) report someone would bring them food while isolating.

- Food insecurity is a major threat: in the past week, $40 \%$ of women and $37 \%$ of men skipped a meal, with $76 \%$ of those individuals reporting that the skipped meal was due to COVID-19. The biggest need reported that was not being addressed, $42 \%$ of respondents said food, with more women mentioning this need than men (45\% vs 35\%).

- Despite large economic losses, assistance to adults in Wajir is limited. Less than $1 \%$ of respondents reported receiving assistance for any reason from any institution.

\section{Knowledge, Attitudes, and Perceived Risk of Infection}

Between July 16-30th, a COVID-19 KAP phone survey was completed with 1,322 adults in Wajir, Kenya. The average age was 44 years, and $68 \%$ were female. Households were part of the ongoing Adolescent Girls Initiative - Kenya cohort across 79 villages in Wajir County. Wajir County is in Northeastern Kenya along the border with Somalia. It is a drought-prone, pastoralist, sparsely populated and under-developed area of Kenya. As at the end of July 2020, there were 28 confirmed cases of COVID-19, largely those who travelled to Somalia and returned to Wajir.

- Overall, respondents in Wajir have mixed knowledge of COVID-19 symptoms and transmission. Only $61 \%$ of respondents know that anyone can become infected with COVID-19, and a substantial percentage believethe myths that COVID-19 is just a common cold (32\%) or is a punishment from God (37\%) which raises some concerns about how and what information is beingshared.

- Prevention: Most adults (84\%) correctly identified handwashing as a method to prevent the spread of COVID-19and over half identified wearing masks (64\%)and standing 1 to 2 meters apart (55\%) as prevention methods.

- Quarantine: Most adults (80\%) know that quarantine facilities are

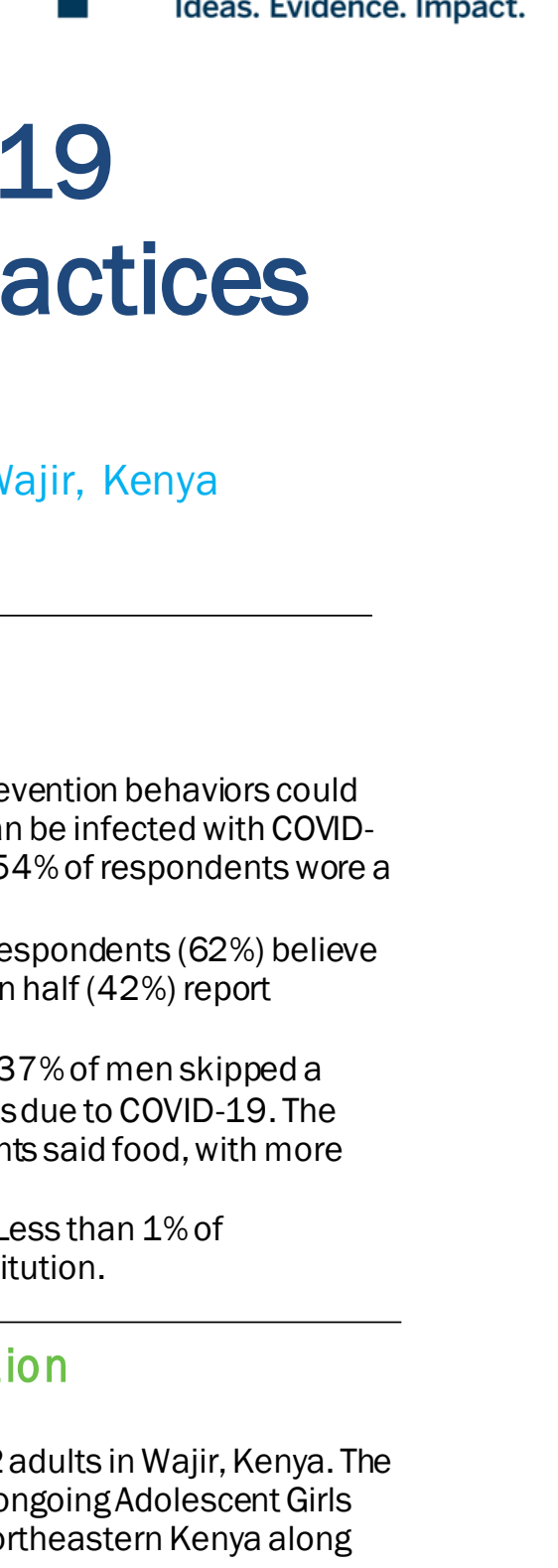
designed for those who have tested positive for COVID-19, yet only a third (33\%) know where the nearest quarantine facility is and only $13 \%$ know that those who have been in contact with someone who has been infected should be in quarantine. There was little consensus on where 
individuals with asymptomatic COVID-19 infection should go to recover, with $47 \%$ saying a health facility, $35 \%$ an isolation facility, and $14 \%$ at home.

- Stigma: Most respondents believed that if they became infected, people would stop talking to them (62\%), stop visiting their house or business (79\%), and gossip about them (72\%). Less than half believed peoplewould bring them needed food (42\%) or medicine (31\%). Compared to women, men reported higher perceived potential stigma and less anticipated social support if they were to become infected. Even after recovery from COVID-19, many respondents feared they would be avoided (40\%), not welcomed back to their mosques (20\%), and not welcomed back by theirfamilies (19\%).

- Experience with COVID-19: Few respondents (1\%) reported knowing someone who has tested positive for COVID-19 or was suspected of being positive.

- Overall, few adults (4\%) consider themselves to be at high risk of COVID-19 infection.

- Among those who have low perceived risk (55\%), the main reasons are: God protects me (57\%) and carrying out recommended prevention behaviors, including social distancing (25\%), wearinga mask (24\%), washing hands (21\%), staying home (21\%), and not travelling (21\%).

\section{CoVID-19 Prevention Methods}

\section{Social Distancing / Wearing Face Masks}

- Both men and women reported interacting with others less than before the COVID-19 pandemic began. Overall, $65 \%$ are avoiding public transportation more, $67 \%$ are seeing friends less, and $45 \%$ are seeing family less.

- Approximately half of respondents (54\%) worea face mask in the last week. The main reasons given for not wearing a mask are that masks are uncomfortable (26\%) and not affordable (18\%).

\section{Handwashing}

- Only 1 in 4 respondents report that there are handwashingstations in public areas near their home, and $72 \%$ do not have a designated place in their home to wash hands. However, nearly all respondents have access to water (96\%) and soap (90\%) for handwashing.

- $70 \%$ say they wash hands after using the toilet and $73 \%$ before eating, which were the common pre-COVID-19 recommendations for handwashing. Fewer respondents wash their hands after coming home from a public place (38\%) or caring for sick people (11\%). Women report handwashing at critical COVID-19 prevention moments significantly more often than men.

\section{Impact of the COVID-19 M itigation Response}

The COVID-19 pandemic and associated social distancing and lockdown measures have had a large economic, health, and social impact on respondents. Many are earning less income due to COVID-19 even as costs of household items have risen, and a substantial percentage report experiencing food insecurity. Relative to need, very few adults are receiving assistance in the form of cash, vouchers, or essential items.

Income/Job loss

- 1 in 5 respondents (20\%) report losing complete or partial income due to COVID-19. Overall, $52 \%$ of respondents report earning less in the past month than they earned before the pandemic began. Meanwhile, $61 \%$ report that the cost of household expenses has increased.

\section{Food Security and Assistance}

- Many participants have been skipping meals or eating less since the COVID-19 pandemic began. In the past 7 days, $40 \%$ of women and $37 \%$ of men skippeda meal, with $76 \%$ of those individuals reporting that the skipped meal was due to COVID-19 disruptions.

- $69 \%$ say they skipped a meal on a couple days last week and $8 \%$ say they skipped every day.

- When asked what their biggest need is that's not being addressed, $42 \%$ of respondents said food, with more women mentioning this need than men (45\% vs 35\%). 
- Less than $1 \%$ of respondents report receiving assistance for any reason, far lower than the $21 \%$ of Nairobi informal settlement residents who received assistance in April 2020.

\section{Health and Safety}

- A small number of respondents (4\%) are not accessing needed health services since the COVID19 pandemic began, putting them at high risk for poor health outcomes. Of that $4 \%$, the main reasons are that they are scared they will be infected with COVID-19 at a clinic (32\%) and cannot afford the cost (23\%).

\section{Women and Children:}

\section{Women are disproportionately bearing the brunt of the pandemic}

- Women are more likely than men to report they are doing more cooking (51\% vs. $20 \%$ ), fetching water ( $40 \%$ vs $21 \%)$, cleaning ( $51 \%$ vs. $18 \%$ ), and childcare (58\% vs. $34 \%$ ) since the pandemic began. They are also more likely to be skipping meals or eating less since the pandemic began.

Children are vulnerable

- While almost all households had an in-school child pre-COVID-19, only $2 \%$ of respondents say their children are learning online and $6 \%$ say children are accessing lessons on the television or radio during the school closures.

- Nearly a third (31\%) say their children have skipped meals or eaten less due to COVID-19.

- Children who have left school or begun working to support their families or selves are particularly vulnerable: $2 \%$ of both male and female children have been working to help earn money for their families.

\section{Conclusions and Recommendations}

COVID-19 public education campaigns in Wajir should continue with a focus on:

- Ensuring that people know that anyone can become infected with COVID-19 and you can spread COVID-19 without showing symptoms.

- Addressingstigma and ensuring that those who test positive for COVID-19 receive needed food and medical care.

Address the health, economic, and social impacts of lockdowns:

- Government and NGO assistance to adults in Wajir has been minimal despite large numbers forgoing meals and experiencing com plete or partial income loss. Assistance in the form of food, vouchers, or cash should be provided and targeted to those with the greatest need.

- As school closures continue, the government must seek out additional, non-technology-based avenues for children to access learning content, as only $2 \%$ of children are learning online and $6 \%$ over television or radio. They will need to ensure that children are re-enrolled in school after the long closures to prevent child marriage and loss of the recent gains in enrollment in the county.

The Population Council conducts research and delivers solutions to improve lives. As COVID-19 spreads around the world, the Population Council is working to lessen the health, social, and economic impact of the outbreak. On the ground in 50 countries and 14 offices, our experts are partnering with governments to develop approaches for rapid research, data collection, monitoring, and mapping, and to test and evaluate behavioral interventions.

Suggested citation: Population Council. 2020. “Wajir, Kenya: Adult COVID-19 Perceptions, Prevention Practices, and Impact. Responses from the first round of adult data collection in Wajir, Kenya"

(C) 2020 The Population Council, Inc. 